\title{
The effect of atherogenic plasma index on collateral development in patients with chronic coronary total occlusion
}

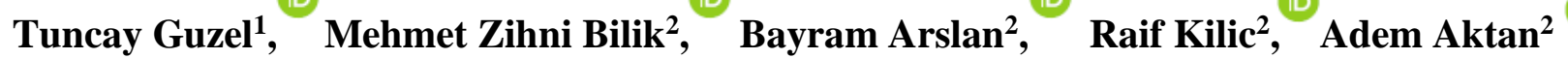
${ }^{I}$ Department of Cardiology, Health Science University, Gazi Yaşargil Training and Research Hospital, Diyarbakır, Turkey ${ }^{2}$ Department of Cardiology, Dicle University, Faculty of Medicine, Diyarbaktr, Turkey

\section{ABSTRACT}

Aim: To demonstrate the correlation between coronary collateral circulation (CCC) and atherogenic plasma index (AIP), one of the factors associated with the formation of collateral vessels.

Methods: Hospital records of patients with chronic total occlusion (100\% stenosis) in at least one coronary artery were evaluated. Triglycerides, HDL level, triglyceride/HDL ratio and atherogenic plasma index before coronary angiography were assessed for the 451 patients who met the study criteria.

Results: Comparison of the two groups in terms of laboratory findings showed that triglyceride/HDL ratio (5.04 \pm 3.13 vs $3.56 \pm 2.12, p<0.001)$ and AIP $(0.63 \pm 0.25$ vs $0.48 \pm 0.25, p<0.001)$ were higher with statistical significance in the weak collateral group. The ROC analysis revealed an association between weak collateral formation and atherogenic plasma index with $64.7 \%$ sensitivity and $66.2 \%$ specificity using a cut-off value of 0.58 for AIP. Accordingly, low AIP was found to be an independent predictor of good collateral artery formation.

Conclusion: This study suggests that a high atherogenic plasma index may be an independent factor associated with poor collateral formation.

Key words: Coronary artery, occlusion, collateral vessels, atherogenic plasma index, triglyceride/HDL ratio.

$\triangle$ Dr. Tuncay Guzel

Department of Cardiology, Health Science University, Gazi Yaşargil Training and Research Hospital, Diyarbakır, Turkey

E-mail: drtuncayguzel@gmail.com

Received: 2021-05-02 / Revisions: 2021-06-14

Accepted: 2021-06-23 / Published online: 2021-10-01

\section{Introduction}

Atherosclerosis constitutes the base of coronary artery disease and is a progressive process with systemic involvement and is accompanied by inflammation [1]. Chronic total occlusion (CTO) of the coronary arteries is defined as the complete obstruction of the vessel lumen for at least three months. In the coronary angiography series, CTO was found in at least one coronary artery in approximately $1 / 3$ of patients. Revascularisation of CTO lesions by percutaneous coronary intervention has positive impacts on the improvement of left ventricular function. Coronary collateral circulation (CCC), which exists in the normal heart, may be defined as vascular structures formed as a chronic, adaptive response between portions of the same coronary artery, or between different coronary arteries, to maintain the perfusion and viability of myocardial tissue distal to the lesion. This ensures blood flow to the ischemic myocardial tissue when critical stenosis or total occlusion disrupts blood flow [2]. Although it was initially accepted that all coronary collateral vessels develop when 
needed, it is currently believed that coronary collateral vascular development occurs both by the formation of new vascular structures (angiogenesis) with the budding of capillaries from existing vascular structures, and by the development of arteriogenesis, which results from the growth and development of anastomotic channels present between the coronary arteries. These two mechanisms occur parallel to each other. When there are cardiovascular risk factors, such as hypercholesterolemia, advanced age, hypertension, diabetes, genetic predisposition, obesity and smoking, the release of angiogenic factors decreases [3]. Therefore, it is considered that the development of CCC is also adversely affected. Collateral arteries often become visible angiographically in cases where more than $90 \%$ of the main coronary artery diameter is occluded. Different methods have been used to show and group CCC angiographically. Rentrop classification classified the collateral area by the filling of the vascular structure, while Gibson and Werner classified the area by the size of the collateral vessel. The mechanism of development of coronary collateral circulation is still unclear, and clinical studies on this subject are ongoing. There is a relationship between factors such as hypertension, age, diabetes mellitus, hyperlipidemia, obesity and systemic inflammatory events, which have impacts on the development of coronary collateral and the inflammatory response caused by lipid parameters in metabolic syndrome and ischemic heart disease. Past studies have shown that vascular endothelial cells are an important factor in the development and progression of CCC $[4,5]$. Myocardial hypoxia may facilitate the development of coronary collateral vessels. Patients with chronic obstructive pulmonary disease (COPD) have chronic hypoxemia.
Ramazan Topsakal et al. showed that the development of coronary collateral vessels in 98 COPD patients was better in patients with COPD than in patients without COPD [6]. JanErik Guelker et al. showed that the triglyceride (TG)/high-density lipoprotein (HDL) ratio can be an independent factor for complex CTO lesions [7]. The logarithm of the molar ratio of TG to high-density lipoprotein cholesterol (HDL-C) levels, the atherogenic index of plasma (AIP), is closely related and inversely proportional to the diameter of low-density lipoprotein cholesterol (LDL-C) particles, which indirectly reflects small dense lowdensity lipoprotein (sdLDL) levels [8]. This value has been established as an index for predicting plasma atherosclerosis and CAD $[9,10]$. Our study was aimed at investigating the effect of AIP obtained by the logarithmic transformation of the TG/HDL ratio on the development of coronary collateral, regardless of other factors, and to determine the relationship between them in patients in whom CCC was found.

\section{Materials and methods}

\section{Study population}

Our study was approved by the Ethics Committee of Dicle University Faculty of Medicine on 21.01.2019 with file number 43. Later in our study, the data of the patients were retrospectively reviewed in the coronary angiography series performed between 03/2014-11/2018 at the Department of Cardiology of the Heart Hospital of Dicle University Faculty of Medicine. The inclusion criteria of the study will basically be based on the criterion of having lesion or lesions assessed as chronic total occlusion (100\% stenosis) in at least one or more coronary arteries in the coronary angiography. Patients were selected consecutively. The exclusion criteria of the 
study include admission to the hospital as myocardial infarction with ST-segment elevation, a history of previous coronary artery bypass, using a statin, being under the age of 18 years, using nonsteroidal or steroid, a history of chronic rheumatological disease, presence of hematological malignancy or any other history of malignancy.

\section{Blood samples and echocardiography}

Blood samples were collected from anterior forearm in all patients included in the study in the supine position after hospitalization in our cardiology clinic. Measurements of lipid levels were performed in serum separated by centrifuge at $3000 \mathrm{rpm}$ at room temperature. Total cholesterol (TC), triglyceride (TG), highdensity cholesterol (HDL) were assessed by Konelab kits and Konnelab 60i and Thermo Clinical Labsystems devices (Thermo Clinical Labsystems Oy Ratostic 2, Vantae, Finland). The LDL cholesterol level was calculated using the Friedewald formula in patients. Left ventricular ejection fractions were measured by transthoracic two-dimensional echocardiography in the echocardiography laboratory during the admission to the Department of Cardiology (Vivid S6, GE Medical Systems, USA).

\section{Coronary angiography}

Selective coronary angiography was performed by the Judkins technique, using $6 \mathrm{~F}$ or $7 \mathrm{~F}$ catheters using the right or left femoral or radial approach in patients. Coronary angiography images were assessed by two experienced cardiologists blinded to the laboratory values and clinical characteristics of the patients. The degree of obstruction in the coronary arteries was decided based on the projection with the greatest stenosis. Collateral development was assessed according to the Rentrop grading. In the evaluation made according to the Rentrop grading; Rentrop 0: No collateral filling,
Rentrop 1: Barely detectable collateral flow and no filling of epicardial arteries, Rentrop 2: Partial filling, contrast medium enters but fails to completely opacify the epicardial arteries, Rentrop 3: Complete perfusion, contrast medium completely opacifies the epicardial arteries. Rentrop 0 and 1 were evaluated as poorly developed collateral circulation, while Rentrop 2 and 3 were considered to be welldeveloped collateral circulation.

\section{Measurement of atherogenic index of plasma (AIP)}

Triglyceride and HDL values were measured from the blood samples collected before the coronary angiography of the patients in the study and were recorded in $\mathrm{mg} / \mathrm{dL}$. The atherogenic index of plasma (AIP) was calculated as the logarithmic transformation of the triglyceride/HDL ratio. AIP of $<0.11$ was considered low risk, AIP of 0.11-0.21 moderate risk, AIP of $>0.21$ increased risk $[8,11]$.

\section{Statistical analysis}

All analyzes were performed with SPSS version 25.0 (SPSS Inc., Chicago, Illinois, USA). Whether the distribution of continuous variables is normal was determined by Kolmogorov-Smirnov and Shapiro-Wilk tests. Descriptive statistics were expressed as mean \pm standard deviation for continuous variables, and as the number of cases and (\%) for the nominal variables. Continuous variables with normal distribution were compared using the Student t-test, and continuous variables with non-normal distribution were compared using the Mann Whitney-U test. Chi-square test and Fisher's Exact test were used to compare categorical variables. Pearson's correlation test was used to evaluate the relationship between normally distributed parameters and Spearman's rho correlation test was used to examine the relationship between parameters that are not normally distributed. In the Pearson 
correlation analysis, the correlation coefficient (r) was calculated. Those with a correlation coefficient between 0 and 0.25 were defined as very weakly correlated, those with a correlation coefficient between 0.25 and 0.50 as weakly correlated, those with a correlation coefficient between 0.50 and 0.69 as moderately correlated, those with a correlation coefficient between 0.70 and 0.89 as strongly correlated, and those with a correlation coefficient between 0.90 and 1 as very strongly correlated.

Also, the significance tests of the correlations were performed and p-values, also known as the degree of significance, were given. The statistical significance level of the obtained data was interpreted with the " $p$ " value. $p$ values of $<0.05$ were considered statistically significant.
Multivariate logistic regression analysis was used to evaluate independent determinants influencing collateral development. All significant variables were included in the logistic regression model, and the results were obtained in the $95 \%$ confidence interval and expressed as odds ratio (OR).

In addition, ROC analysis and curves (Receiver Operating Characteristics Curve) were used to determine the diagnostic performance and cutoff values of the TG/HDL ratio and the Atherogenic index of plasma (AIP).

\section{Results}

A total of 451 patients, 126 women (27.9\%) and 325 men $(72.1 \%$ ), aged between 33 and 92 years old, with a mean age of $61.75 \pm 11.16$

Table 1. Demographic and clinical characteristics of the patients.

\begin{tabular}{|c|c|c|c|c|}
\hline \multicolumn{2}{|c|}{$\begin{array}{c}\begin{array}{c}\text { Demographic and clinical } \\
\text { characteristics }\end{array} \\
\end{array}$} & $\begin{array}{c}\text { Poor collateral } \\
\mathrm{N}=\mathbf{2 3 2} \\
\end{array}$ & $\begin{array}{c}\text { Good collateral } \\
\mathbf{N}=219 \\
\end{array}$ & $p$ \\
\hline \multicolumn{2}{|l|}{ Age } & $60.70 \pm 10.85$ & $62.86 \pm 11.40$ & 0.039 \\
\hline \multirow{2}{*}{ Gender, $(\mathrm{N}, \%)$} & Female & $69(29.7 \%)$ & $57(26.0 \%)$ & \multirow{2}{*}{0.380} \\
\hline & Male & $163(70.3 \%)$ & $162(74.0 \%)$ & \\
\hline \multicolumn{2}{|l|}{$\mathrm{HT}, \mathrm{S}(\mathrm{N}, \%)$} & $98(42.2 \%)$ & $77(35.2 \%)$ & 0.123 \\
\hline \multicolumn{2}{|l|}{$\mathrm{DM}, \mathrm{S}(\mathrm{N}, \%)$} & $105(45.3 \%)$ & $55(25.1 \%)$ & 0.000 \\
\hline \multicolumn{2}{|c|}{ Smoking, S (N, \%) } & $127(54.7 \%)$ & $57(26.0 \%)$ & 0.000 \\
\hline \multicolumn{2}{|c|}{ Family history, S (N, \%) } & $28(12.1 \%)$ & $15(6.8 \%)$ & 0.059 \\
\hline \multicolumn{2}{|c|}{ Previous MI, S (N, \%) } & $57(24.6 \%)$ & $49(22.4 \%)$ & 0.583 \\
\hline \multicolumn{2}{|c|}{ Beta-blocker, S (N, \%) } & $74(31.9 \%)$ & $59(26.9 \%)$ & 0.249 \\
\hline \multicolumn{2}{|c|}{ ACE-I/ARB, S (N, \%) } & $94(40.5 \%)$ & $76(34.7 \%)$ & 0.203 \\
\hline \multicolumn{2}{|l|}{$\mathrm{CCB}, \mathrm{S}(\mathrm{N}, \%)$} & $47(20.3 \%)$ & $33(15.1 \%)$ & 0.143 \\
\hline \multicolumn{2}{|l|}{ Nitrate, S (N, \%) } & $21(9.1 \%)$ & $18(8.2 \%)$ & 0.753 \\
\hline \multicolumn{2}{|l|}{ ASA, S (N, \%) } & $39(16.8 \%)$ & $40(18.3 \%)$ & 0.685 \\
\hline \multicolumn{2}{|c|}{ Clopidogrel, S (N, \%) } & $10(4.3 \%)$ & $5(2.3 \%)$ & 0.230 \\
\hline
\end{tabular}

HT: Hypertension, DM: diabetes mellitus, MI: myocardial infarction, ACE-I/ARB: Angiotensin-converting enzyme inhibitor/ Angiotensin receptor blockers, CCB: calcium channel blocker, ASA: acetylsalicylic acid. 
years, were included in our study. The mean age of the patients was found to be $60.21 \pm 10.83$ years in men and $65.72 \pm 11.05$ years in women. 219 patients (48.6\%) with Rentrop grade 2 and 3 collaterals angiographically constituted the good collateral group and 232 patients $(51.4 \%)$ with grade 0 and 1 collateral the poor collateral group. Those with good collaterals and those with poor collaterals were compared regarding gender, age, diabetes mellitus (DM), hypertension (HT), smoking, family history, previous myocardial infarction (Pre. MI), use of Angiotensin-converting enzyme inhibitor (ACE-I), Angiotensin receptor blockers (ARB), acetylsalicylic acid (ASA), clopidogrel, calcium channel blocker (CCB), nitrate, and beta-blocker (Table 1).

Considering the previous histories of the patients, $105(45.3 \%)$ patients of the poor collateral group and $55(25.1 \%)$ patients of the good collateral group had a history of DM. When both groups were compared, there was a statistically significant difference $(p<0.001)$. $127(54.7 \%)$ patients in the poor collateral group and $57(26.0 \%)$ patients in the good collateral group had a history of smoking. Statistically significant difference was found between the two groups $(p<0.001)$.

Angiographic findings of patients according to collateral grade after coronary angiography are shown in (Table 2). Comparing the arteries developing poor collaterals angiographically, while chronic total occlusion (CTO $\geq 2$ vessels) was found in the LAD in $36 \%$, in the CX in $22.8 \%$, in the RCA in $56 \%$, and in multiple vessels in $14 \%$, chronic total occlusion was found in $32 \%$ in the $\mathrm{LAD}$, in $22 \%$ in the $\mathrm{CX}$, in $63 \%$ in the RCA, in $14 \%$ in multiple vessels in the good collateral group. However, there was no statistically significant difference between the two groups $(p>0.05)$. Comparing all three coronary arteries, generally more CTOs were
Table 2. Angiographic findings of the patients.

\begin{tabular}{|l|l|l|l|}
\hline $\begin{array}{l}\text { Angiographic } \\
\text { findings }\end{array}$ & $\begin{array}{l}\text { Poor } \\
\text { collateral } \\
\text { N=232 }\end{array}$ & $\begin{array}{l}\text { Good } \\
\text { collateral } \\
\text { N=219 }\end{array}$ & $\boldsymbol{p}$ \\
\hline LAD & $84(36 \%)$ & $71(32 \%)$ & 0.397 \\
\hline Cx & $53(23 \%)$ & $47(22 \%)$ & 0.724 \\
\hline RCA & $129(56 \%)$ & $137(63 \%)$ & 0.133 \\
\hline CTO $\geq 2$ vessels & $32(14 \%)$ & $30(14 \%)$ & 0.977 \\
\hline
\end{tabular}

LAD: Left anterior descending artery, Cx: Circumflex artery, RCA: Right coronaryartery, CTO: Chronic total occlusion.

detected in the RCA. This result was consistent with previous large-scale studies. Comparing the poor collateral group and the good collateral group in terms of CTOs in the RCA, although good collaterals were seen more, there was no statistically significant difference $(p=0.133)$.

Although high-density lipoprotein (HDL) level was higher in the good collateral group, there was no statistically significant difference ( $p=0.397)$. Fasting glucose level, triglyceride level, and total cholesterol level were significantly higher in the poor collateral group ( $p<0.001, p<0.001, \quad p=0.019$, respectively). The sodium level was significantly higher in the good collateral group, incidentally $(p=0.009)$ (Table 3).

When two groups were compared in terms of echocardiographic findings, the mean left ventricular ejection fraction $(\mathrm{EF})$ at admission was $48.05 \pm 7.96$ in the poor collateral group and $51.24 \pm 9.77$ in the good collateral group and there was a statistically significant difference between the two groups $(p<0.001)$ (Table 3).

When two groups were compared regarding the triglyceride/HDL ratio (TGHDL), it was found to be $5.04 \pm 3.13$ in the poor collateral group 
Table 3. Laboratory and echocardiographic findings of the patients.

\begin{tabular}{|c|c|c|c|}
\hline $\begin{array}{c}\text { Laboratory and } \\
\text { Echocardiographic Findings }\end{array}$ & $\begin{array}{c}\text { Poor Collateral } \\
\quad \mathrm{N}=\mathbf{2 3 2}\end{array}$ & $\begin{array}{c}\text { Good Collateral } \\
\mathrm{N}=\mathbf{2 1 9}\end{array}$ & $p$ \\
\hline $\mathrm{WBC}\left(\mathrm{x} 10^{3} \mu \mathrm{L}\right)$ & $9.40 \pm 2.50$ & $9.13 \pm 2.56$ & 0.262 \\
\hline $\mathrm{RBC}\left(\mathrm{x} 10^{6} \mu \mathrm{L}\right)$ & $4.96 \pm 0.62$ & $4.96 \pm 0.80$ & 0.976 \\
\hline HGB $(\mathrm{g} / \mathrm{dL})$ & $13.84 \pm 2.05$ & $13.82 \pm 2.18$ & 0.956 \\
\hline HCT & $42.66 \pm 5.25$ & $42.44 \pm 5.75$ & 0.676 \\
\hline $\operatorname{MCV}\left(x 10^{3} \mu \mathrm{L}\right)$ & $86.20 \pm 6.24$ & $86.41 \pm 5.94$ & 0.722 \\
\hline RDW & $12.22 \pm 1.96$ & $12.20 \pm 1.87$ & 0.952 \\
\hline Glucose (mg/dL) & $158.5 \pm 75.60$ & $132.3 \pm 55.23$ & 0.000 \\
\hline Urea $(\mathrm{mg} / \mathrm{dL})$ & $40.97 \pm 22.50$ & $42.75 \pm 23.23$ & 0.410 \\
\hline Kreatinin (mg/dL) & $1.03 \pm 0.74$ & $1.09 \pm 0.89$ & 0.424 \\
\hline Total Protein (gr/dL) & $7.24 \pm 0.82$ & $7.87 \pm 0.65$ & 0.328 \\
\hline Albumin (gr/dL) & $3.71 \pm 0.42$ & $3.65 \pm 0.42$ & 0.140 \\
\hline Total Bilirubin (mg/dL) & $0.65 \pm 0.35$ & $0.66 \pm 0.33$ & 0.933 \\
\hline ALT (U/L) & $24.96 \pm 19.55$ & $25.19 \pm 20.93$ & 0.906 \\
\hline $\operatorname{AST}(\mathrm{U} / \mathrm{L})$ & $27.50 \pm 1.44$ & $30.04 \pm 2.05$ & 0.313 \\
\hline ALP (U/L) & $82.09 \pm 28.49$ & $86.23 \pm 31.36$ & 0.175 \\
\hline GGT (U/L) & $37.74 \pm 2.63$ & $41.91 \pm 4.30$ & 0.411 \\
\hline LDH (U/L) & $264.6 \pm 116.6$ & $274.1 \pm 124.4$ & 0.403 \\
\hline Triglyceride (mg/dL) & $186.7 \pm 86.75$ & $143.9 \pm 69.16$ & 0.000 \\
\hline Total Cholesterol (mg/dL) & $183.9 \pm 47.95$ & $173.4 \pm 46.63$ & 0.019 \\
\hline HDL (mg/dL) & $41.77 \pm 26.73$ & $43.38 \pm 8.71$ & 0.397 \\
\hline $\mathrm{LDL}(\mathrm{mg} / \mathrm{dL})$ & $102.1 \pm 39.12$ & $102.4 \pm 38.41$ & 0.935 \\
\hline Calcium (mg/dL) & $9.19 \pm 0.72$ & $9.16 \pm 0.55$ & 0.553 \\
\hline Sodium (mmol/L) & $136.6 \pm 2.73$ & $137.3 \pm 2.94$ & 0.009 \\
\hline Potassium (mmol/L) & $4.47 \pm 0.41$ & $4.44 \pm 0.45$ & 0.527 \\
\hline CRP (mg/dL) & $0.95 \pm 0.12$ & $1.35 \pm 0.24$ & 0.151 \\
\hline TSH (IU/mL) & $1.42 \pm 0.15$ & $1.96 \pm 0.58$ & 0.401 \\
\hline $\mathrm{T} 3(\mathrm{pg} / \mathrm{mL})$ & $4.98 \pm 2.88$ & $4.65 \pm 1.27$ & 0.339 \\
\hline T4 (ng/L) & $17.68 \pm 5.44$ & $16.99 \pm 3.42$ & 0.318 \\
\hline TGHDL & $5.04 \pm 3.13$ & $3.56 \pm 2.12$ & 0.000 \\
\hline AIP & $0.63 \pm 0.25$ & $0.48 \pm 0.25$ & 0.000 \\
\hline $\mathrm{EF}(\%)$ & $48.05 \pm 7.96$ & $51.24 \pm 9.77$ & 0.000 \\
\hline
\end{tabular}

ALT: Alanine transaminase, AST: Aspartate transaminase, ALP: Alkalen fosfataz, GGT: Gama glutamil transpeptidaz, LDH: Laktat dehidrogenaz, HDL: High-density cholesterol. LDL: Low density lipoprotein, CRP: C-reactive protein, TSH: Thyroid-stimulating hormone, TGHDL: High triglyceride high-density lipoprotein cholesterol ratio, AIP: Atherogenic index of plasma, EF: Ejection fraction.

and $3.56 \pm 2.12$ in the good collateral group on average. It was statistically significantly higher in the poor collateral group $(p<0.001)$ (Table 3). When two groups were compared concerning the atherogenic index of plasma
(AIP), it was found to be $0.63 \pm 0.25$ in the poor collateral group, and $0.48 \pm 0.25$ in the good collateral group on average. It was statistically significantly higher in the poor collateral group $(p<0.001)$ (Table 3). 
When correlation analysis was performed between collateral grade and the TG/HDL ratio (TGHDL) $\left(r_{s}=-0.299, p<0.001\right)$ and between collateral grade and the atherogenic index of plasma $\left(r_{s}=-0.299, p<0.001\right)$, there was a weak negative correlation which was statistically significant ( $\mathrm{r}_{\mathrm{s}}$ : Spearman's rho correlation coefficient) (Table 4).

Table 4. Correlation analysis between collateral grade and the TG/HDL ratio (TGHDL) and the atherogenic index of plasma (AIP).

\begin{tabular}{|l|c|c|}
\hline Parameter & $\mathbf{r}_{\mathrm{s}}$ & $\boldsymbol{p}$ \\
\hline TG/HDL & -0.299 & 0.000 \\
\hline AIP & -0.299 & 0.000 \\
\hline
\end{tabular}

In the multivariate logistic regression analysis performed to find the factors affecting collateral development, the TG/HDL ratio (TGHDL) and the atherogenic index of plasma (AIP) were found to be statistically significant, independently of other factors. Accordingly, low TG/HDL ratio (TGHDL) and AIP were found to be independent predictors of the development of the good collateral arteries $(p<0.001)$ (Table 5).

Table 5. Multivariate Logistic Regression Analysis between collateral development and the TG/HDL Ratio (TGHDL) and the atherogenic index of plasma (AIP).

\begin{tabular}{|l|c|c|c|}
\hline Parameter & OR & $\mathbf{9 5 \%}$ CI & $\boldsymbol{p}$ \\
\hline Age & 1.008 & $0.987-1.029$ & 0.470 \\
\hline Gender & 0.631 & $0.383-1.038$ & 0.070 \\
\hline TG/HDL & 0.819 & $0.741-0.905$ & 0.000 \\
\hline AIP & 0.083 & $0.035-0.196$ & 0.000 \\
\hline
\end{tabular}

In the ROC analysis of the data, the development of collateral was compared with the TG/HDL ratio (TGHDL) and the atherogenic index of plasma (AIP). There was a statistically significant negative correlation between collateral development and the TG/HDL ratio (TGHDL) and the atherogenic index of plasma (AIP). When the cutoff value was considered 3.81 for the TG/HDL ratio and 0.58 for the atherogenic index of plasma (AIP), the increase in the TG/HDL ratio and the atherogenic index of plasma was found to be associated with the presence of poor collaterals with a sensitivity of $64.7 \%$ and specificity of $66.2 \%$ (Table 6, Figure 1).

\section{Discussion}

The factor affecting coronary collateral development is an inflammatory response. One of the factors that started this inflammatory process is AIP. When starting our study, we aimed to investigate the predictability of a decrease in collateral development when there is an increase in AIP and the relationship between them. In order to reveal this relationship, patients were selected from the groups that showed homogenous distribution in terms of factors other than AIP, which we think is particularly effective on collateral development. Since we aimed to investigate the effect of AIP on CCC in patients with coronary artery disease, we did not include patients taking lipid-lowering drugs in our study. Therefore, in our study, the possible effects of drugs with the ability to reduce lipid levels on HDL cholesterol, CCC and triglyceride levels were not mentioned. In our study, the left ventricular systolic functions were statistically significantly higher in the good collateral group, and a statistically significant correlation was found between AIP, diabetes mellitus, smoking and collateral development. In a study conducted by Abaci et al. on 205 diabetes mellitus (DM) patients, it was shown that collateral vessel development in DM was worse than in non-diabetic patients. We can say that 
Table 6: ROC analysis.

\begin{tabular}{|l|l|l|l|l|l|l|}
\hline \multicolumn{7}{|c|}{ Area Under the Curve [AUC] } \\
\hline \multirow{2}{*}{} & AUC & $p$ value & $\begin{array}{l}\text { 95\% Confidence } \\
\text { interval (CI) }\end{array}$ & Cut off value & Sensitivity & Specificity \\
\cline { 3 - 7 } & & Lower-Upper Limit & & \\
\hline TG/HDL & 673 & 0.00 & $623-722$ & 3.81 & $64.7 \%$ & $66.2 \%$ \\
\hline AIP & 673 & 0.00 & $623-722$ & 0.58 & $64.7 \%$ & $66.2 \%$ \\
\hline
\end{tabular}

$\mathrm{DM}$ is an important factor among the factors affecting the development of coronary collateral [12].

For visible collateral vessels, there must be total or subtotal occlusion of the coronary arteries [13]. Therefore, we included patients with at least one total occluded coronary artery in our study. Also, collateral development is a timedependent continuity. The development of coronary collaterals was demonstrated in the first six hours following acute myocardial infarction, and all collaterals developed were found to become visible in the coronary angiography performed within 24 hours after myocardial infarction. To make an accurate assessment of CCC, we did not include patients who underwent coronary angiography in the first 24 hours after acute coronary syndrome. An experimental study showed that HDL-C increases coronary artery blood flow through NO-mediated vasodilation [14]. This finding shows that HDL cholesterol can positively affect collateral development with the increased effect of NO. Rossi et al. have shown that HDL cholesterol is a powerful indicator of the number and function of circulating endothelial progenitor cells (EPCs), which also repair the endothelial layer [15]. Spieker et al. showed that HDL cholesterol is an endothelial protective factor in a hypercholesterolemic man without any risk factors for coronary artery

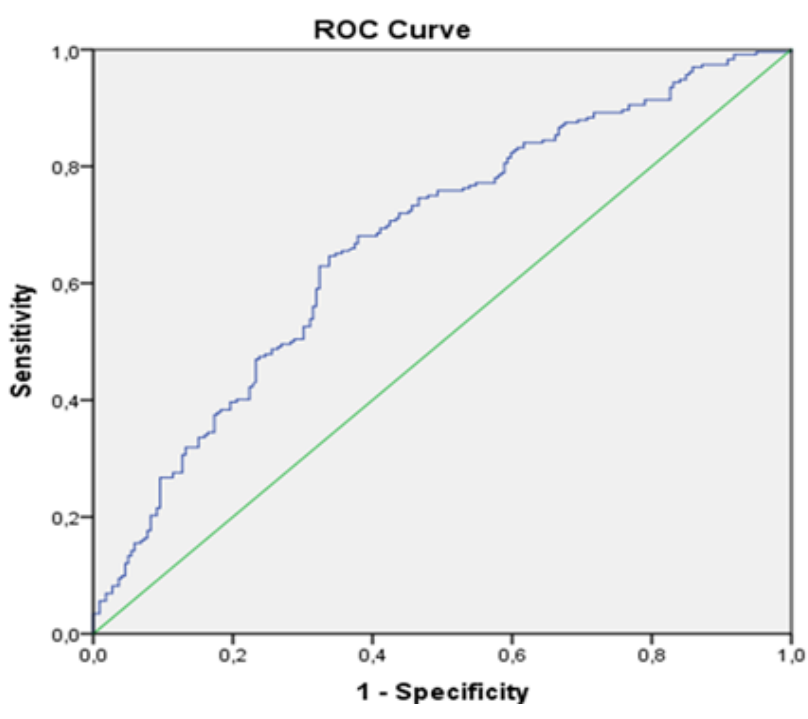

Figure 1: ROC analysis comparison of the relationship between collateral development and the atherogenic index of plasma and the TG/HDL Ratio.

disease [16]. In healthy individuals without an additional risk factor for cardiovascular disease, low HDL-C has been shown to be an independent risk factor for endothelial dysfunction [17].

The results obtained from these studies clearly prove that HDL-C has endothelial layer protection and anti-inflammatory effects. Therefore, patients with weak CCC may have low HDL cholesterol levels. This can be explained by the anti-inflammatory and endothelial protective properties of HDL-C. However, in our study, although HDL cholesterol levels were found to be higher in the 
group developing good collaterals, no statistically significant difference was found $(p$ $=0.397$ ).

First described by Dobiášová and Frohlich in 2001, AIP [18] is a comprehensive lipid index and a powerful marker for predicting CAD risk [19]. While Niroumand et al. suggested that AIP could be used as a regular monitoring index of CAD in daily practice [20], Wan et al. proved an elevated AIP to be a strong independent predictor of all-cause mortality and cardiovascular disease after coronary revascularization [21]. However, several recent studies have proven AIP to be of significant value in assessing the severity of coronary syndrome. Another study showed a significant relationship between AIP and the progression of coronary artery calcification [22]. Furthermore, in a 7-8-year follow-up study of 2,676 middle-aged adults, AIP was confirmed to be a prognostic marker for predicting CADrelated morbidity [23]. AIP is independently associated with the occurrence of CTO and is thought to predict the presence of CTO and disease severity [24].

Nevertheless, a higher AIP may be considered an inflammatory marker that mediates endothelial function and the growth of collateral circulation by affecting a number of inflammatory reactions. Strong evidence in the prediction of the increase in AIP reducing coronary collateral development has provided important data: AIP is a determinant via local inflammatory response.

Our study has some limitations. First, the sample size was relatively small, a multi-center study should be designed to present the result as strong evidence. Secondly, the bias was inevitable as the subjects included as a casecontrol study were enrolled from a single center. Thirdly, as this study was performed cross-sectionally, not knowing clearly whether the blood sample was collected after proper diet preparation and the condition of other daily events affecting the atherogenic index of plasma related to TG/HDL ratio made it difficult to comment on its relationship with the development of CCC. Fourthly, as HDL cholesterol dysfunction may also occur, serum HDL cholesterol level does not fully demonstrate the function of HDL cholesterol. Fifthly, it is unknown whether the results of our study can be extended to other populations.

\section{Conclusion}

We demonstrated that a high atherogenic plasma index is an independent factor associated with weak collateral formation. The atherogenic index of plasma, which is simple, non-invasive, and economic, obtains a fast result and has an important value, should be carefully evaluated in determining individuals at risk for cardiovascular diseases, especially in individuals with a family history of coronary heart diseases. The development of cardiovascular diseases may be decreased by reducing the atherogenic index of plasma.

Funding: The author(s) received no financial support for the research, authorship, and/or publication of this article.

Conflict of Interest: The authors declare that they have no conflict of interest.

\section{Ethical statement:}

The study was approved by Local Clinical Research Ethics Committee (Date and Decision Number: 2019.01.21-43), and written informed consent was obtained from each subject.

\section{Open Access Statement}

This is an open access journal which means that all content is freely available without charge to the user or his/her institution under the terms of the Creative Commons Attribution NonCommercial License (http://creativecommons.org/licenses/by- 
nc/4.0). Users are allowed to read, download, copy, distribute, print, search, or link to the full texts of the articles, without asking prior permission from the publisher or the author.

Copyright (c) 2021: Author (s).

\section{References}

[1]Ross R. Atherosclerosis--an inflammatory disease. N Engl J Med. 1999;340(2):11526.

[2]Cohen MV. The functional value of coronary collaterals in myocardial ischemia and therapeutic approach to enhance collateral flow. Am Heart J. 1978;95(3):396404.

[3]Waltenberger J. Impaired collateral vessel development in diabetes: potential cellular mechanisms and therapeutic implications. Cardiovasc Res. 2001;49(3):554-60.

[4]Monette JS, Hutchins PM, Ronsein GE, et al. Patients with coronary endothelial dysfunction have impaired cholesterol efflux capacity and reduced HDL particle concentration. Circ Res. 2016;119(1):83-90.

[5]Kris-Etherton PM, 吕ris WS, Appel LJ. Fish consumption, fish oil, omega-3 fatty acids, and cardiovascular disease. Circulation. 2002;106(21):2747-57.

[6]Topsakal R, Eryol NK, Abaci A, et al. The relation between chronic obstructive pulmonary disease and coronary collateral vessels. Angiology. 2005;56(6):651-56.

[7]Guelker JE, Bufe A, Blockhaus C, et al. The atherogenic index of plasma and its impact on recanalization of chronic total occlusion. Cardiol J. 2020;27(6):756-61.

[8]8. Teixeira BC, Lopes AL, Macedo RCO, et al. Inflammatory markers, endothelial function and cardiovascular risk. J Vasc Brasil. 2014;13(2):108-15.

[9]Tonet AC, Karnikowski M, Moraes CF, et al. Association between the-174G/C promoter polymorphism of the interleukin-6 gene and cardiovascular disease risk factors in Brazilian older women. Braz J Med Biol Res. 2008;41(1):47-53.

[10] Tousoulis D, Plastiras A, Siasos G, et al. Omega-3 PUFAs improved endothelial function and arterial stiffness with a parallel antiinflammatory effect in adults with metabolic syndrome. Atherosclerosis. 2014;232(1):10-16.

[11] Toikka JO, Ahotupa M, Viikari JS, et al. Constantly low HDLcholesterol concentration relates to endothelial dysfunction and increased in vivo LDLoxidation in healthy young men. Atherosclerosis. 1999;147(1):133-38.

[12] Abacı A, Oğuzhan A, Kahraman S et al. Effect of Diabetes Mellitus on Formation of Coronary Collateral Vessels. Circulation. 1999;99(17):2239-42.

[13]Elayda MA, Mather VS, Hall RJ, et al. Collateral circulation in coronary artery disease. Am J Cardiol. 1985;55(1):58-60.

[14]Levkau B, Hermann S, Theilmeier G, et al. High-density lipoprotein stimulates myocardial perfusion in vivo. Circulation. 2004;110(21):3355-59.

[15] Rossi F, Bertone C, Montanile F, et al. HDL cholesterol is a strong determinant of endothelial progenitor cells in hypercholesterolemic subjects. Microvasc Res. 2010;80(2):274-79.

[16] Spieker LE, Sudano I, Hurlimann D, et al. High-density lipoprotein restores endothelial function in hypercholesterolemic men. Circulation. 2002;105(12):1399-402.

[17]Higashi Y, Matsuoka H, Umei $\mathrm{H}$, et al. Endothelial function in subjects with isolated low HDL cholesterol: role of nitric oxide and circulating progenitor cells. Am J 
Physiol Endocrinol Metab.

2010;298(2):E202-9.

[18]Dobiášová M, Frohlich J. The plasma parameter log (TG/HDL-C) as an atherogenic index: correlation with lipoprotein particle size and esterification rate inapob-lipoprotein-depleted plasma (FERHDL). Clin Biochem. 2001;34(7):58388.

[19]Khazaál MS. Atherogenic Index of Plasma (AIP) as a parameter in predicting cardiovascular risk in males compared to the conventional dyslipidemic indices (cholesterol ratios). Karbala J Med. 2013;6(1):1506-11.

[20]Niroumand S, Khajedaluee M, KhademRezaiyan M, et al. Atherogenic Index of Plasma (AIP): A marker of cardiovascular disease. Med J Islam Repub Iran. 2015;29:240.

[21] Wan K, Zhao J, Huang H, et al. The association between triglyceride/highdensity lipoprotein cholesterol ratio and allcause mortality in acute coronary syndrome after coronary revascularization. PLoS One. 2015;10(4):e0123521.

[22]Nam JS, Kim MK, Nam JY, et al. Association between atherogenic index of plasma and coronary artery calcification progression in Korean adults. Lipids Health Dis. 2020;19(1): 157.

[23] Onat A, Can G, Kaya H, et al. Atherogenic index of plasma ( $\log 10$ triglyceride/highdensity lipoprotein-cholesterol) predicts high blood pressure, diabetes, and vascular events. J Clin Lipidol. 2010;4(2):89-98.

[24]Liu T, Liu J, Wu Z, et all. Predictive value of the atherogenic index of plasma for chronic total occlusion before coronary angiography. Clin Cardiol. 2021;44(4):51825 . 